\title{
Simulation of Geomagnetic Reversals Through Magnetic Critical Models
}

\author{
V. H. A. Dias, J. O. O. Franco, and A. R. R. Papa \\ Observatório Nacional, Rua General José Cristino 77, \\ São Cristóvão, Rio de Janeiro, 20921-400 RJ, BRAZIL \\ and \\ Universidade do Estado do Rio de Janeiro, \\ Rua São Francisco Xavier 524, Maracanã, \\ Rio de Janeiro, 20550-900 RJ, BRAZIL
}

Received on 29 September, 2007

\begin{abstract}
We use numerical simulations of a well-known phase-transition model to study reversals of the geomagnetic field. Each ring current in the geodynamo was supposed to behave as a magnetic spin while the magnetization of the model was supposed to be proportional to the Earth's magnetic dipole. We have performed a sizedependence study of the calculated quantities. Power laws were obtained for the distribution of times between reversals. Some of our results are closer to actual ones than the corresponding to previous simulations. For the largest systems that we have simulated the exponent of the power law tends towards values very near -1.5 , generally accepted as the right value for this phenomenon. Some possible trends for future works are advanced.
\end{abstract}

Keywords: Ising model; Reversals; Computer simulation

\section{INTRODUCTION}

During the last decades many research efforts of statistical physicists have been devoted to the study of the earthquake problem [1]. However, it is far from being the unique geophysical subject where statistical physics could bring some light. Let us mention the problems of magnetic storms [2,3] and geomagnetic reversals [4]. We will focus our attention on geomagnetic reversals.

The field produced at the interior of the planet has its main source in a complex system of internal currents that eventually produce geomagnetic reversals. They are periods during which the Earth's magnetic field swaps hemispheres. Many of them have been documented. Periods between geomagnetic reversals present power law distribution functions, which can be the signature of some critical system as the mechanism of their source [5]. There are other mechanisms capable of producing power laws (for example, superposition of some distributions [1], self-organized criticality [6] and non-extensive versions of statistical mechanics [7]) but we will not extend on them upon here. In Fig. 1 we present the sequence of geomagnetic reversals from 80 million years (My) to our days. The data was obtained from the most recent and complete record that we have found $[8,9]$. Fig. 2 presents the distribution of periods between reversals, it follows a power law,

$$
f(t)=c t^{d}
$$

where $f(t)$ is the frequency distribution of periods between consecutive reversals, $c$ is a proportionality constant and $d$ is the exponent of the power-law and the slope of the graph in $\log$-log plots. For the present case we have approximately -1.5 as slope value.

The magnetic field needs a finite time to change its direction (the time between the moment at which the dipolar component is no more the main one and the moment at

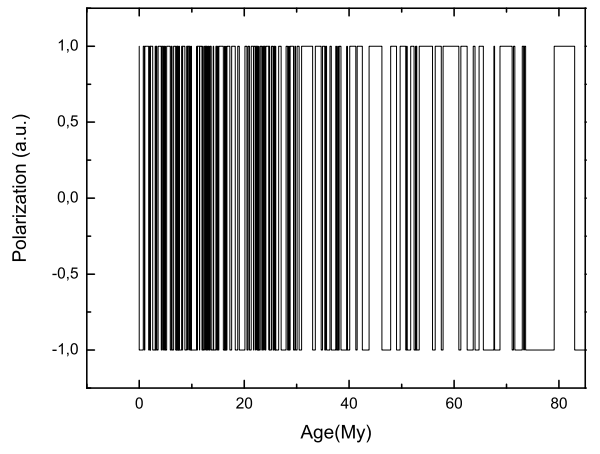

FIG. 1: Representation of geomagnetic reversals from around $80 \mathrm{My}$ ago to our days. We arbitrarily have assumed -1 as the current polarization. We have not plotted the period from $80 \mathrm{My}$ to $165 \mathrm{My}$ (for which also a record exists) for the sake of clarity. There was a period with no reversals from 80 to $120 \mathrm{My}$ (the beginning appears in the plot). The period from $120 \mathrm{My}$ to $165 \mathrm{My}$ was very similar to the period from 0 to $40 \mathrm{My}$ (in number of reversals and in the average duration of reversals). The data was obtained from Cande and Kent $[8,9]$.

which it is again the main one but building up in the opposite direction). This time is in the average around 5000 years. It is small if compared to the smallest interval between reversals already detected ( 10,000 years) and to the average interval between reversals. There are clustered periods of high activity from 40 My ago to our days and during the period 165-120 My (not shown), a period of low activity between 80 and 40 My ago and a period of almost null activity from 120 to 80 My.

The sequence of Earth's magnetic field reversals seems to be a non-equilibrium process. One can infer this from Fig. 1 where the average time interval between successive reversals 


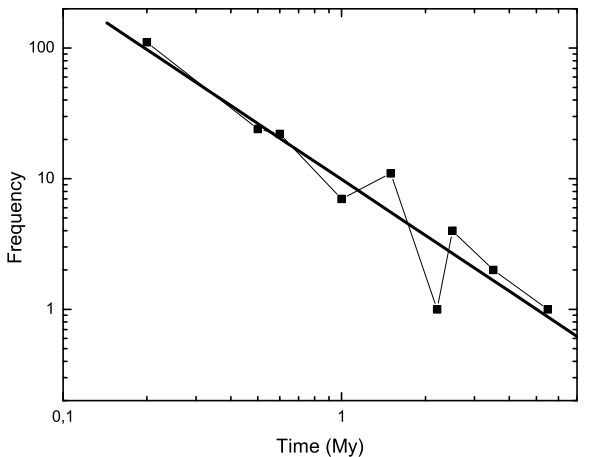

FIG. 2: Frequency distribution of periods between reversals from 80 My to our days. The slope of the solid line is around -1.5 . The data was obtained from Cande and Kent [8,9].

seems to increase with geological time. Unfortunately, the reversal series is unique and relatively small, there is no other similar record (for earthquakes, for example, exists a record for each fault, and from the Moon obtained during the Apollo program).

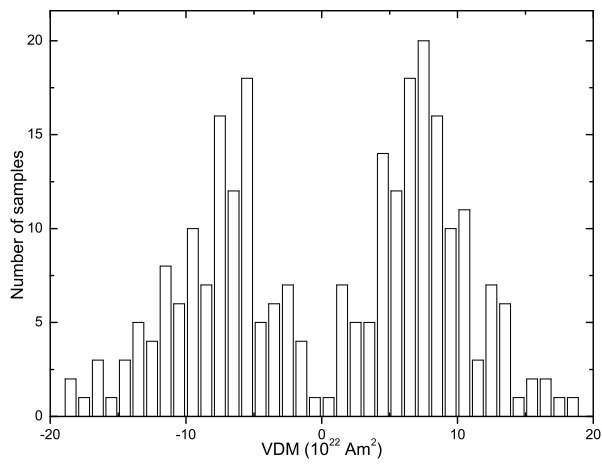

FIG. 3: Distribution of virtual dipole moment (VDM) values for samples not older than $10 \mathrm{My}$. Negative values of VDM correspond to reverse polarization and are represented by 119 samples. Positive values of VDM represent normal polarization and are represented by 142 samples. The data was obtained from Kono and Tanaka [10].

Contrary to the record of reversals (known from $160 \mathrm{My}$ up to nowadays) the record of magnetic field intensities exists just for around $10 \mathrm{My}$ [10]. Fig. 3 shows the distribution of Earth's dipole values for the last $10 \mathrm{My}$. The distribution of dipole values for actual reversals (Fig. 3) follows a function between a bi-normal and a bi-log-normal distribution. However, the number of experimental points is also small and there will certainly be changes in these facts when new measurements become available.

Geomagnetic reversals have called a great attention since their discovery and this interest remains nowadays. Works devoted to the study of the time distribution of geomagnetic reversals include, among many others, statistical studies on reversals distributions and correlations [5,11-13], modelling of the problem [14-16] and experimental studies [8,9,1719]. Gaffin [11] pointed out that long-term trends and non-stationary characteristics of the record could hamper a formal detection of chaos in geomagnetic reversal record. It is our opinion that actually it is very difficult to detect in a consistent manner that geomagnetic reversals present any systematic characteristic at all, without mattering which this characteristic could be (including chaos). Its nature remains an open question.

In this work we use the 2D Ising model near $T_{c}$ to mimic the Earth's magnetic field and its reversals. Ising systems have already been used to simulate reversals of the Earth magnetic field [16]. Our work differs from it in that while they used the Q2R automata we have used a regular Metropolis updating algorithm around the critical temperature. Some of our results also differ essentially from them and are closer to actual ones.

The study of geomagnetic reversals can contribute to the comprehension of important processes in Earth's evolution. This includes from the internal dynamics of the planet to processes involving the evolution of living organisms. The Earth is continuously bombarded by charged particles mainly born at the Sun. Large fluxes of those particles are incompatible with life. Fortunately, only a tiny fraction of them hits the Earth's surface. The most part is deviated by the geomagnetic field. However, during reversals the field intensity decreases drastically which means a more intense bombarding. The "magnetic shield" is reduced. Those increases in the flux of particles could have interfered in life cycles of the planet.

\section{THE MODEL}

Simulation of the core by three-dimensional magnetohydrodynamic equations is an extremely difficult task $[14,15]$. To diminish the number of degrees of freedom an alternative way is to use $\mathrm{N}$ coupled disk dynamos models [20]. Following Seki and Ito [16] we further simplified the last one by associating spins to currents.

The Ising model is some kind of paradigm among magnetic models for condensed matter because it is the simplest one that presents a phase transition at temperatures different from zero (in $D>1$, where $\mathrm{D}$ is the dimension). But Ising models have found applications in areas away from solid-state physics, for example, in immunology [21], stock market theory [22] and earthquake dynamics [23], among many others. In this work we use the 2D Ising model to simulate the Earth's dynamo, particularly the reversals of the Earth's magnetic field.

Our motivation for using the Ising model to simulate the Earth's dipole comes from the relative success of a previous work by Ito and Seki [16] and, at the same time, from the 
fact that the dipole suffers reversals, in a similar way to the Ising model near the critical temperature. From the practical point of view the motivation comes from the possibility of generate good synthetic data with simple models to diminish the problem of limited availability of experimental data.

In absence of external field the Hamiltonian $H$ for the Ising model is:

$$
H=-J \sum_{i, j} s_{i} s_{j}
$$

where the sum runs over all neighbor pairs, $s_{i}$ and $s_{j}$ are spins and $J$ is the interaction constant between spins. The critical temperature of the Ising system for $2 \mathrm{D}$ is $T_{c}=2.269$ in units of $J / k_{B}$, where $k_{B}$ is the Boltzmann constant. By definition, each spin $s_{i}$ can assume only two values or orientations: 1 and -1 . It is worth to mention that the Ising model was introduced by Wilhelm Lenz in 1920 and exactly solved for one dimension by his student Ernest Ising, in 1925, and in two dimensions by Lars Onsager, in 1944. It was shown that for 2D there is a phase transition.

In our model we assume, following Seki and Ito [16], that each spin represents a current ring in the Earth's dynamo (i.e., that turbulent eddies behave as magnetic spins).

The magnetization $M$ for the model is defined as:

$$
M=\frac{\sum_{i=1}^{N} s_{i}}{N}
$$

where the sum runs over all the spins and $N$ is the total number of spins. It can take values between -1 and 1 . It is assumed in our model that the magnetization represents the geomagnetic dipole.

Now we call the attention to the fact that the magnetic moment of eddies are of vectorial nature (they are not restricted to values +1 or -1 ) and, in principle, they exist in a broad range of sizes (limited uniquely by the core size). Later will be clear that in ferro-magnetic-like simulations domains of spins with the same orientation can be considered to represent larger eddies while, in anti-ferrimagnetic-like simulations this distinction have to be imposed by us. On the other hand, the idea of neighbor eddies is customarily used in the study of such phenomena (we refer the interested reader to the book by Merril et al. [4]).

Many efforts have been made to enlarge the size of simulated Ising models when trying to represent systems of physical or biological nature [24]. The reason is simple: the numbers of elements in condensed matter physics and biological systems are usually huge, around $10^{23}$ and $10^{14}$, respectively. Physical models for the Earth's magnetic field are mostly based in a small number of adequately connected dynamos. Using small 2D Ising systems to simulate their interaction and behavior must sound a natural choice. We have performed simulations on 2D square Ising systems at temperatures near $T_{c}$ and studied some of the relevant characteristics of the distribution in time of magnetization reversals (transitions between states of positive and negative magnetization). We have applied periodic boundary conditions [25]. At the same time we have studied the size dependence of distributions. We have used $L x L$ systems where $L=5,10,20,30,40$ and 50 .

We believe that the Ising model near $T_{c}$ could be a good model for reversals of the Earth's magnetic field because of the critical character present in the Ising model and suggested by figures 2 and 3 (see below). The resulting magnetic field of the Earth is the superposition of several components (dipolar, quadripolar, and so on). When we simulate reversals through 2D Ising systems we are mainly focusing on the dipolar moment (which is the main component the most part of the time). In this way, we are able to mimic the essentially 3D Earth by a $2 \mathrm{D}$ Ising system. The rotation of the Earth imposes a preferential direction to the possible orientations of magnetic moments associate to eddies. From both dynamo-like and convectionlike approaches, the structures in the core are of tubular type with symmetry axes parallel to the rotation axis of the Earth. At the same time, those structures traverse the core from the bottom to the top (top meaning the "superior" hemisphere and bottom meaning "inferior" hemisphere). This facts support the simulation of a $3 \mathrm{D}$ system by a $2 \mathrm{D}$ one. The magnetic field, however, never reaches the value "zero". It diminishes to values very near zero in other directions than the north south one.

\section{RESULTS AND DISCUSSION}

\section{A. Ito and Seki simulations}

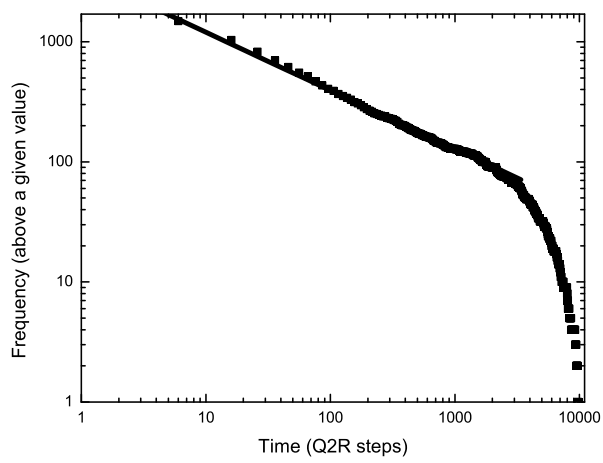

FIG. 4: Frequency distribution inter-reversal periods (Ito and Seki model). The solid line slope is -0.5 which means -1.5 for a normal (not above-a-given-value) histogram, as in Fig. 2.

We started by reproducing the simulations by Ito and Seki. The two main results are shown in Figs. 4 and 5. Fig. 4 presents the distribution of periods between reversals. The distribution follows a power-law with around -1.5 for the slope value. In Fig. 5 we present the distribution of magnetization values. There are two approximately symmetric peaks (like in the actual distribution, Fig. 3) and a valley around the zero magnetization value (like in real measurements also). How- 


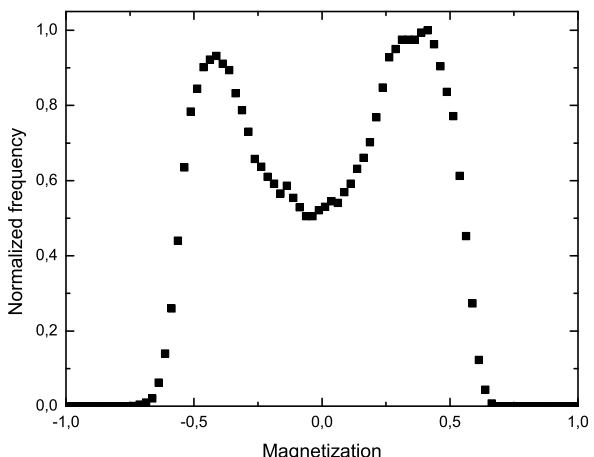

FIG. 5: Frequency distribution of magnetization values (Ito and Seki model). Note that the central valley is around 50 percent of the peaks highs while for actual measurements (Fig. 3) it is less than 10 percent.
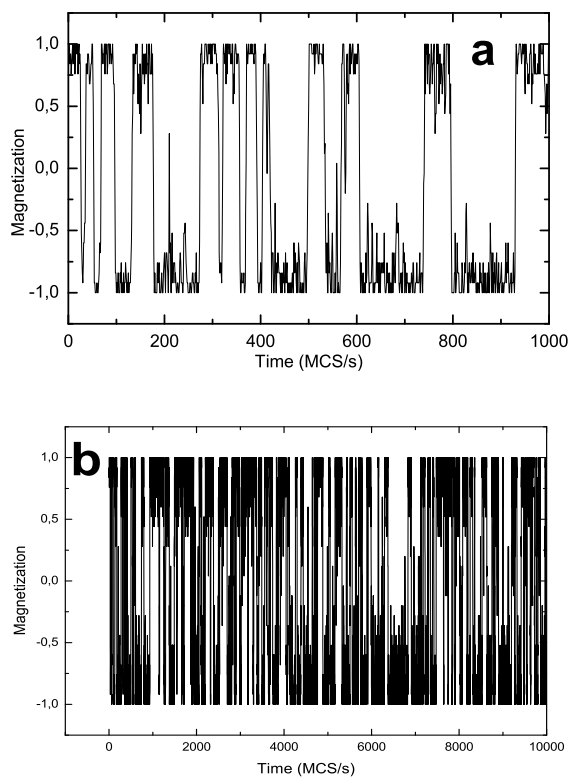

FIG. 6: Time dependence of the magnetization for temperatures slightly below $T_{C}(T=2.26)$. a) A sample of $1000 \mathrm{MCS} / \mathrm{s}$ pertaining to a long run. b) A longer sample (up to $10000 \mathrm{MCS} / \mathrm{s}$ ). Both cases correspond to a $5 \times 5$ system size.

ever, we note that the relative high of the valley is much more higher than for actual measurements (Fig. 3).

\section{B. Ferromagnetic Metropolis simulations}

Next, we have simulated the Ito and Seki model using instead of the Q2R a Metropolis updating algorithm. In Fig. 6 we show some typical time dependence of magnetization for temperatures slightly below $T_{c}$. They qualitatively resemble the results presented in Figs. 3 and 5 of reference [16]. However, they are quantitatively different from them (see below). Fig. 7 shows the result of eliminating all the fluctuations in Fig. 6b. We have only marked the jumps through the value zero and assigned a value 1 to all the positive values and -1 for the negative ones. Note the pictorial and qualitative similarities between Figs. 1 and 7. In particular, note that Fig. 7 also presents clustered periods of greater activity (between 7000 and $10000 \mathrm{MCS} / \mathrm{s}$ ) and of lower activity (between 6000 and $7000 \mathrm{MCS} / \mathrm{s}$ and around $1000 \mathrm{MCS} / \mathrm{s}$, for example).

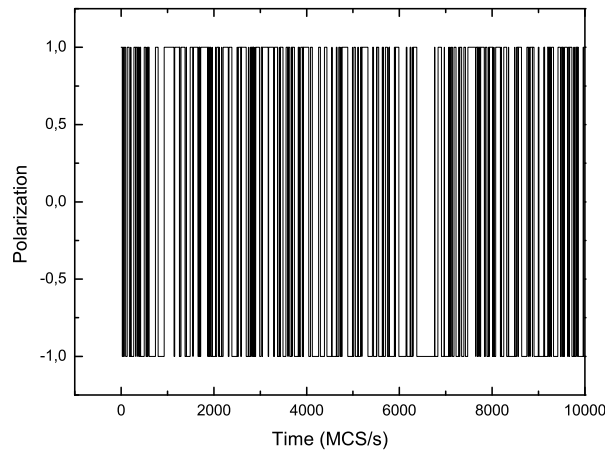

FIG. 7: A binary version of the dependence in Fig. 2b. It was assigned a value 1 to all values $M>0$ and a value of -1 for all values $M<0$. Note the similarity with Fig. 1 where actual reversals are presented.

Figure 8 shows the distribution of intervals between consecutive reversals $(\mathrm{T}=2.26)$ for different system sizes. The bold straight line is a guide for the eye in all cases. The distribution follows a power law. However its apparition presents a size dependence. For larger periods the distribution follows an exponential. The exponential dependence can easily be confirmed by representing the data in a semi-log graph (not shown). For $5 \times 5$ systems the exponential occupies almost all the histogram interval (Fig. 8a) while for systems of size $50 \times 50$ it is almost inexistent (Fig. 8c). We have not found the initial power law dependence in the literature but in our simulations and in the work by Seki and Ito [16]. This is a quite remarkable fact given that the Ising model has been analytically and computationally (very extensively) explored during many years. In Fig. 9 it is shown the size dependence of the power-law exponent for a fixed temperature $T=2.26$ (very near $T_{c}$ ). Data is satisfactorily fitted by an exponential function. The results for the slope value range from -2.0 (for $5 \times 5$ systems) to -1.55 (for $50 \times 50$ systems). As can be seen, the larger the system the closer to the most accepted value (-1.5) for the slope on actual reversals distribution [4].

We show in Fig. 10 the distribution of magnetization values $(\mathrm{T}=2.26)$ for $5 \times 5$ and $50 \times 50$ systems. For the smallest system that we have simulated, maximum occurs at the extreme values $M= \pm 1$. For larger systems they occur at intermediate values. While for small systems, fluctuations can drive the system to absolute values of magnetization near 1 , and 

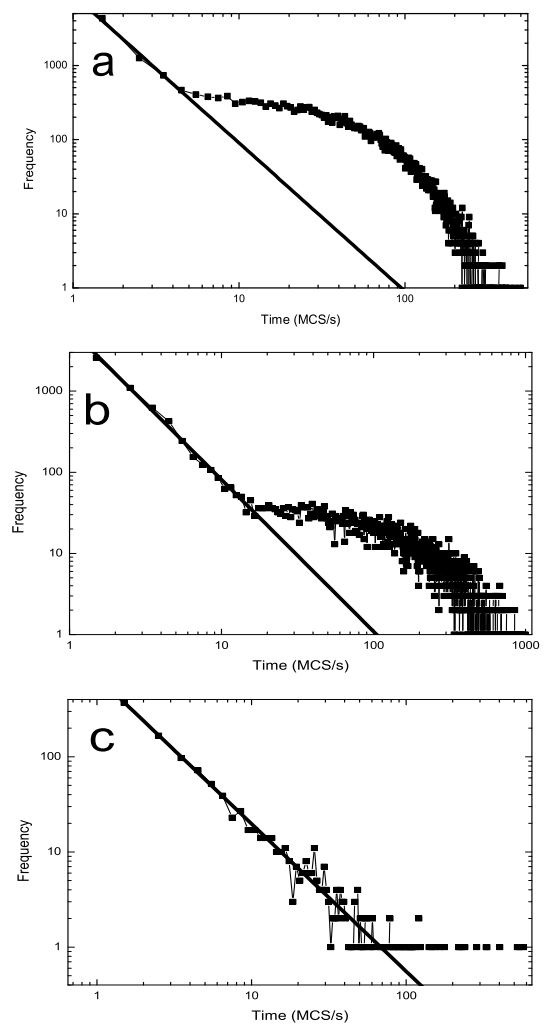

FIG. 8: Distribution of periods between consecutive reversals for our

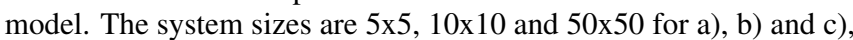
respectively. In all cases the bold straight line is a guide for the eye. Slopes values are a) -2.0 , b) -1.87 and c) -1.55 . Runs were done at a fixed $T=2.26$.

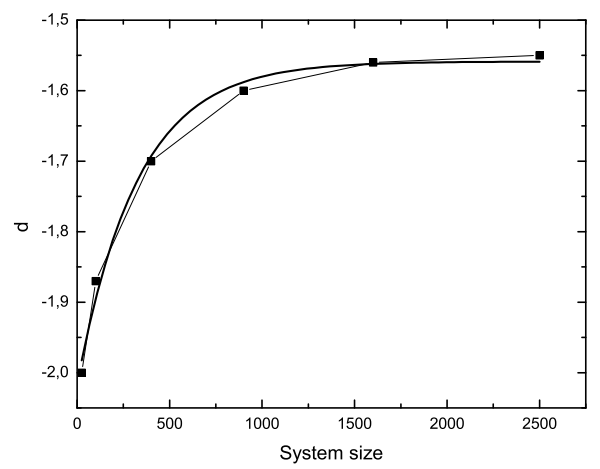

FIG. 9: Size dependence for the slope of the distribution of periods between consecutive reversals in our model. The bold curve is an exponential fit to data. It has the form $y=y_{0}+A \cdot \exp (-x / t)$, where $y_{0}=-1.559 \pm 0.014, A=-0.457 \pm 0.023$ and $t=326 \pm 49$.

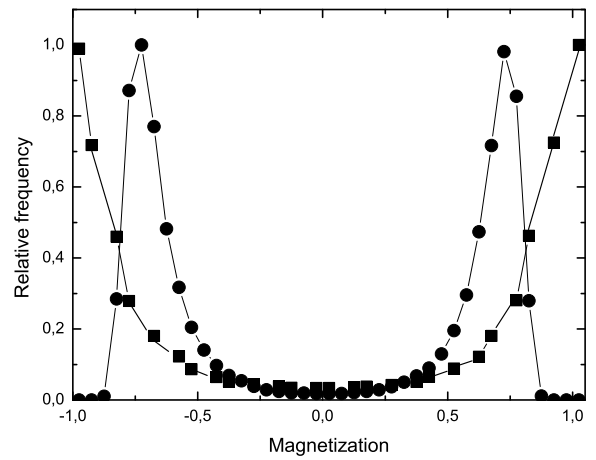

FIG. 10: Distributions of magnetization values. For $5 \times 5$ systems (squares) the maxima appear at the extremes. For larger systems, and in particular for 50x50 systems (circles), maxima appear at values of magnetization $|M| \neq 1$. The valley value is around 10 percent of the peaks values, closer to actual measurements than Ito and Seki simulations.

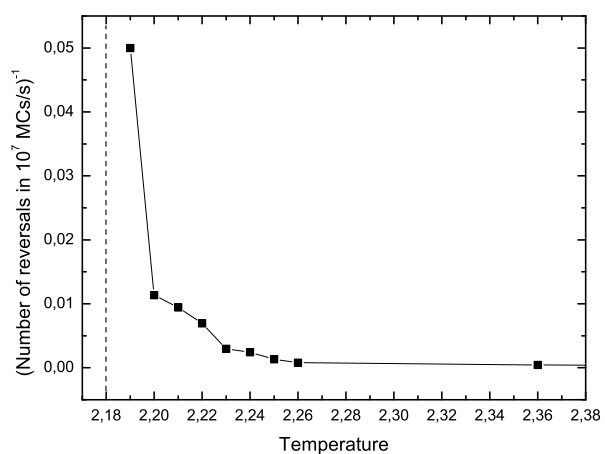

FIG. 11: Temperature dependence of the mean time interval between consecutive reversals. For temperatures below 2.18 there were no reversals (for simulations up to $10^{6} \mathrm{MCS} / \mathrm{s}$ ).

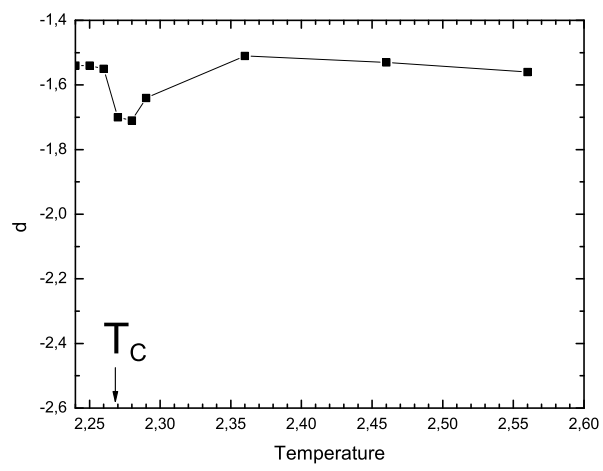

FIG. 12: Temperature dependence of the power-law exponent. A system size 50x50 was used in all cases. 


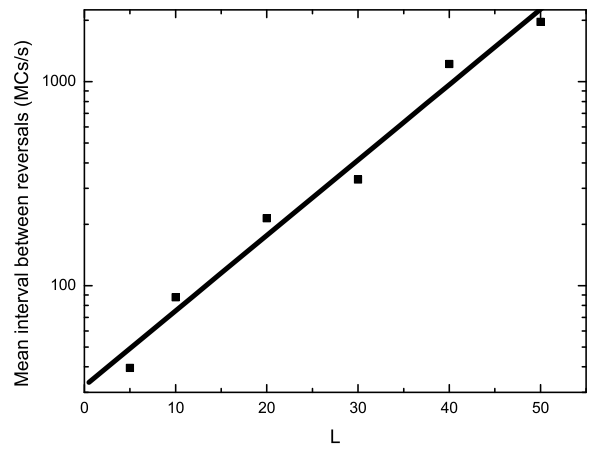

FIG. 13: Size dependence of the mean time interval between consecutive reversals. The same temperature $T=2.26$ was used in all cases. The straight bold line is an exponential fit $\sim \exp ($ const.L) (for systems $L x L$ ).

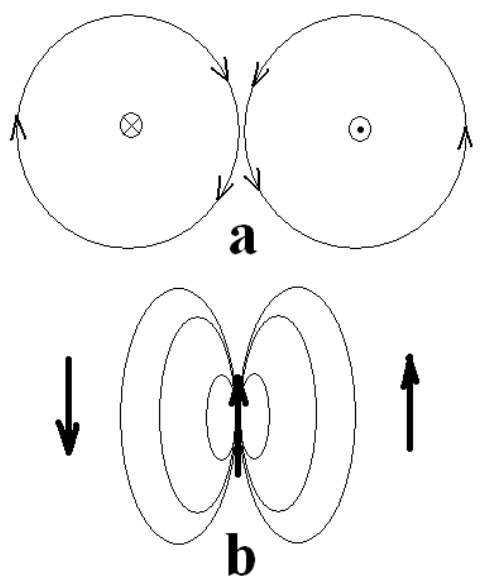

FIG. 14: Schematic representation of the a) mechanical; and b) magnetic; interaction between idealized neighbor eddies. In a) we present a view from above (perpendicular to the plane containing eddies). In b) we present a side view; the central dipole interacs favorably with the left one.

there are not counterparts, for larger systems, a fluctuation to an absolute value near 1 in a part of the system will be accompanied almost always by a value far from those values in other pieces of the system. Like in [16] the distributions follow very roughly a bi-normal function. However, in a previous work [26] on block distribution functions for the Ising model in 2D, 3D and 4D the test was done formally and it was clear that they do not obey gaussians distributions. In this particular our simulations are superior to those by Seki and Ito [16]. We have reproduced their simulations and obtained that, while in our simulations (and in actual reversals) the distribution highs for zero magnetization is around 0.1 of the maximum, for Q2R simulations it is around 50 percent. This difference is probably associated to the

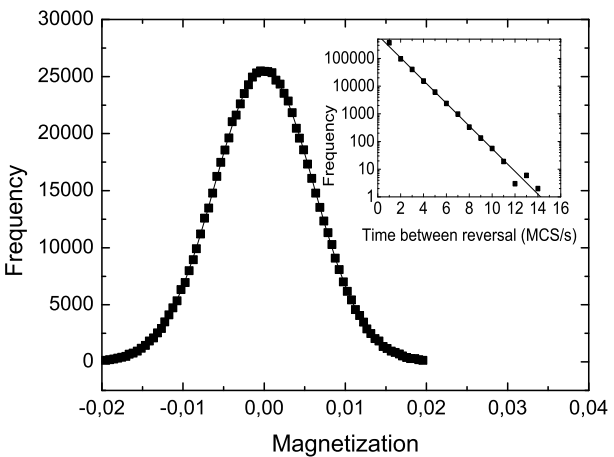

FIG. 15: Frequency distribution of magnetization values (Ising, antiferromagnetic, Metropolis). The magnetization is not a good order parameter, its distribution around zero is a gaussian. The inset shows the distribution of periods between reversals. Note that it is presented in a semi-log plot, so the line is not a power-law but an exponential.

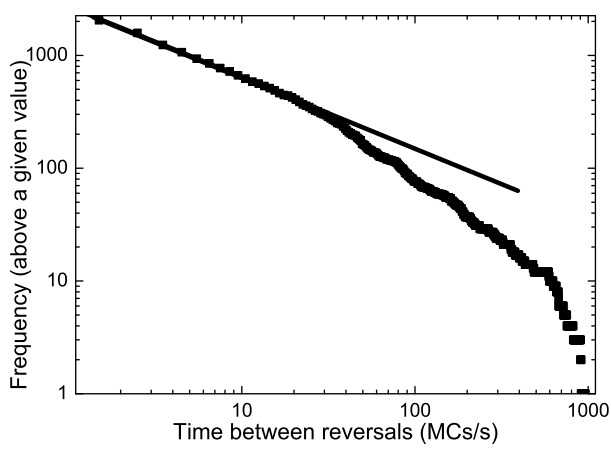

FIG. 16: Frequency distribution of inter-reversal periods (Ising, antiferrimagnetic, Metropolis). The solid line has a slope value around -0.5 .

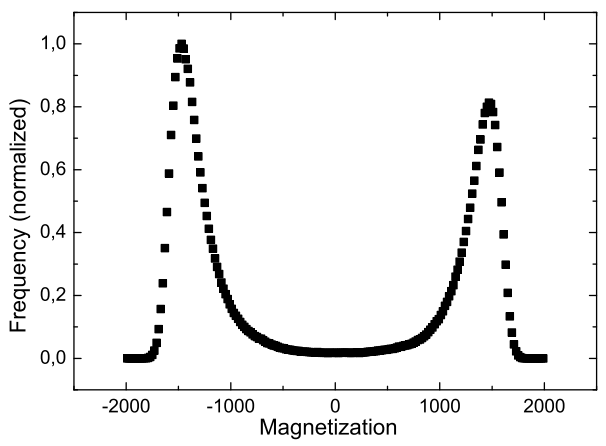

FIG. 17: Frequency distribution of magnetization values (Ising, antiferrimagnetic, Metropolis). Once more, the valley high is closer to actual measurements than for the Ito and Seki model. 
non-ergodic character of Q2R simulations.

Figure 11 shows the dependence of the average duration of time intervals between consecutive reversals on temperature (always for temperatures near the critical point). These results can help us to understand the way in which external factors affect the reversal regime. For more intense perturbations (high temperatures) the reversals are more frequent. For less intense perturbations (low temperatures) there is a lower number of reversals for time unit.

In our simulations the temperature plays the role of a tuning parameter. For temperatures well above $T_{c}$ we observe only small fluctuations around $M=0$, for temperatures well below $T_{c}$ we observe a state of constant magnetization values 1 or -1 with seldom flips to -1 or 1 values, respectively. We have studied the dependence of the power-law slope on temperature for temperatures $\mathrm{T}$ around $T_{c}$ (Fig. 12). Note that for temperatures near $T_{c}$ or above the slope remains almost constant and in a narrow interval (from -1.75 to -1.55). Another point to be highlighted in Figure 12 is that for temperatures above and below $T_{c}$ the physics under magnetization reversals are very different. For temperatures below $T_{c}$ it is essentially a tunnelling through an energy barrier while for temperatures above $T_{c}$ it is well represented by random walks returns to the origin. However, the slope of the inter-reversal interval distribution change in a very gradually manner (at least up to the temperatures that we have simulated).

Figure 13 presents the mean interval between consecutive reversals as a function of the system size. This dependence gives some qualitative insight on which is the volume of Earth's interior that effectively carries the currents that produce the magnetic field (but equally, it could be associated to the number of eddie currents effectively producing the magnetic field). For larger systems reversals are less frequent than for small systems. As expected [27] the data in Fig. 13 can be satisfactorily fitted by an exponential function $\exp ($ const.L) for $L x L$ system sizes.

Despite of the dynamical character of reversals mentioned at the introductory section, there are some pieces of our simulations very similar to the actual sequence of reversals. That is the case in Fig. 7, for example, between Montecarlo steps of numbers around 1000. Probably, some historical ingredient, (i.e., a non-equilibrium approach) could be taken in future attempts to describe the phenomenon. Such an ingredient lacks in our work. Simulations with varying temperature or system size are two of the possibilities opened by the present approach.

\section{Anti-ferromagnetic Metropolis simulations}

Following Ito and Seki we have initially used a ferromagnetic version of the Ising model to simulate the geodynamo.
However, an anti-ferromagnetic type of interaction seems to better represent actual eddies interactions. The magnetic moment of neighbor eddies is more favorable to an anti-ferromagnetic-like arrange both mechanically and magnetically as illustrated in Fig. 14. We have simulated 50x50 2D Ising anti-ferromagnetic systems. (the same Hamiltonian than in Equation (2) except for the minus sign). Unfortunately, the magnetization is not a good order parameter for antiferromagnetic systems and the result for the distribution of magnetization values around zero give a Gaussian as can be seen in Fig. 15. The distribution for periods between reversals is of exponential type (see the inset of Fig. 15).

\section{Metropolis Anti-ferrimagnetic simulations}

An improvement in the model is the introduction of different sizes for spins. We have done this by simulating an anti-ferrimagnetic system (the same Hamiltonian than in Equation (2) except for the minus sign and for the fact that neighbor spins have no more the same value) where it was supposed that the spin size in a sublattice is twice the size of the spin in the other sublattice (raising the critical temperature to the double of lattices where all the spins have the same absolute value).

We present in Fig. 16 the distribution of periods between reversals for 50x50 anti-ferrimagnetic systems. The value near -0.5 for the slope remains (as in Ito and Seki and ferromagnetic models). Our field reversal exponent is closely related to the global persistent exponent [28]. The last one is calculated from simultaneously evolving many systems until the magnetization first change sign and keeping track of the fraction $p(t)$ of surviving systems (systems that have not suffered a reversal up to time $t$ ). Our exponent is calculated from following a single system and recording time intervals between consecutive reversals. It is our believe that they describe essentially the same phenomena.

In Figure 17 the distribution of magnetization values for the same system is shown. Once more two peaks are present as well as a valley with less than 10 percent of peaks' highs. Comparison of results in figures like Fig. 17 and results in Fig. 3 must be done carefully. Note that the results in Fig. 3 are unbounded (the only possible bound could be the dipole value for the case in which the whole core rotates as a single unit). The usual values seem to be far from this bound. Our results, on the other hand, have a bound \pm 1 imposed by the model. This factor give one possible direction for future works.

\section{CONCLUSIONS}

We have simulated 2D square Ising systems to mimic the time dependence of geomagnetic reversals. It is quite interesting that despite off the different physics involved in temperatures above (random walks returns to the origin) and bellow (tunnelling through an energy barrier) the critical tem- 
perature, the slope of the distribution of times between reversals has a smooth dependence on temperature. This fact will eventually need a deeper study. There is a dependence of the mean interval between reversals in both temperature and system size. However, as shown, all the studied properties reach stationary values for relatively small systems, which permits to use the temperature as the unique free parameter. There are two points to be highlighted: first, that as in our simulations the actual distribution of intensities has a deep valley (amplitude approximately 0.1 of the maximum) for magnetization values near zero; and second, that our simulations reproduce the power law dependence of actual reversals (with a slope value very near the actual one). In this sense our simulations (Metropolis) are superior when compared to Q2R simulations.
The necessity of more detailed models including, for example, the fact that there is a continuous scale of eddies is apparent. Some works are currently running along these lines and will be published elsewhere.

\section{Acknowledgments}

The authors sincerely acknowledge partial financial supports from FAPERJ (Rio de Janeiro Founding Agency) and $\mathrm{CNPq}$ (Brazilian Founding Agency). We are indebted to an anonymous reviewer whose criticisms and comments have greatly helped in the final presentation of the work.
[1] D. Sornette, Critical Phenomena in Natural Sciences, (Springer, Berlin, 2004).

[2] A. R. R. Papa, L. M. Barreto, and N. A. B. Seixas, J. Atm. Sol. Terr. Phys., 68, 930 (2006).

[3] Magnetic storms are periods that last from one to three days during which the field suffers rapid variations. (2004), however, they seriously affect many human activities. They are mainly caused by phenomena in the Sun that affect the Earth's atmosphere.

[4] R. T. Merrill, M. W. McElhinny, and P. L. McFadden, The magnetic field of the Earth; Paleomagnetism, the Core, and the Deep Mantle, (Academic Press, 3rd Ed., San Diego, 1998).

[5] A. R. T. Jonkers, Phys. Earth Planet. Int., 135, 253 (2003).

[6] P. Bak, C. Tang, and K. Wiesenfeld, Phys. Rev. Lett. 59, 381 (1987).

[7] M. Gell-mann and C. Tsallis, Nonextensive entropy: interdisciplinary applications, (Oxford University Press, Oxford, 2004).

[8] S. C. Cande and D. V. Kent, J. Geophys. Res. 97, 13917 (1992).

[9] S. C. Cande and D. V. Kent, J. Geophys. Res. 100, 6093 (1995).

[10] M. Kono and H. Tanaka, In: The Earth's Central Part: Its structure and Dynamics, (T. Yukutabe, Ed. Terrapub, Tokyo, 1995).

[11] S. Gaffin, Phys. Earth Planet. Int. 57, 284 (1989).

[12] C. Constable and C. Johnson, Phys. Earth Planet. Int. 153, 61 (2005).

[13] M. Cortini and C. Barton, J. Geophys. Res. 99, 18021 (1994).
[14] P. D. Mininni, A. G. Pouquet, and D. C. Montgomery, Phys. Rev. Lett. 97, 244503 (2006).

[15] P. D. Mininni and D. C. Montgomery, Phys. Fluids 18, 116602 (2006).

[16] M. Seki and K. Ito, J. Geomag. Geoelectr. 45, 79 (1993).

[17] R. Zhu, K. A. Hoffman, Y. Pan, R. Shi, and D. Li, Phys. Earth Planet. Int. 136, 187 (2003).

[18] B. M. Clement, Nature 428, 637 (2004).

[19] J.-P. Valet, L. Meynadier, and Y. Guyodo, Nature, 435, 802 (2005).

[20] M. Shimizu and Y. Honkura, J. Geomag. Geoelectr. 37, 455 (1985).

[21] D. Stauffer, Int. J. Mod. Phys. C 5, 513 (1993).

[22] L. R. da Silva and D. Stauffer, Physica A, 294, 235 (2001).

[23] A. Jimenez, K. F. Tiampo, and A. M. Posadas, Nonlin. Processes Geophys. 14, 5 (2007).

[24] D. Stauffer, Physica A 244, 344 (1997).

[25] A. R. R. Papa, Int. J. Mod. Phys. C 9, 881 (1998).

[26] K. Binder, Z. Phys. B - Cond. Mat. 43, 119 (1981).

[27] H. Meyer-Ortmanns and T. Trappenberg, J. Stat. Phys. 58, 185 (1990).

[28] S. N. Majumdar, A. J. Bray, S. J. Cornell, and C. Sire, Phys. Rev. Lett. 77, 3704 (1996). 